\title{
A Regularized Algorithm for the Proximal Split Feasibility Problem
}

\author{
Zhangsong Yao, ${ }^{1}$ Sun Young Cho, ${ }^{2}$ Shin Min Kang, ${ }^{3}$ and Li-Jun Zhu ${ }^{4,5}$ \\ ${ }^{1}$ School of Mathematics \& Information Technology, Nanjing Xiaozhuang University, Nanjing 211171, China \\ ${ }^{2}$ Department of Mathematics, Gyeongsang National University, Jinju 660-701, Republic of Korea \\ ${ }^{3}$ Department of Mathematics and RINS, Gyeongsang National University, Jinju 660-701, Republic of Korea \\ ${ }^{4}$ School of Mathematics and Information Science, Beifang University of Nationalities, Yinchuan 750021, China \\ ${ }^{5}$ School of Management, Hefei University of Technology, Hefei 230009, China
}

Correspondence should be addressed to Sun Young Cho; ooly61@yahoo.co.kr and Shin Min Kang; smkang@gnu.ac.kr

Received 28 April 2014; Accepted 17 June 2014; Published 2 July 2014

Academic Editor: Jong Kyu Kim

Copyright (C) 2014 Zhangsong Yao et al. This is an open access article distributed under the Creative Commons Attribution License, which permits unrestricted use, distribution, and reproduction in any medium, provided the original work is properly cited.

The proximal split feasibility problem has been studied. A regularized method has been presented for solving the proximal split feasibility problem. Strong convergence theorem is given.

\section{Introduction}

Throughout, we assume that $\mathscr{H}_{1}$ and $\mathscr{H}_{2}$ are two real Hilbert spaces, $f: \mathscr{H}_{1} \rightarrow \mathscr{R} \cup\{+\infty\}$ and $g: \mathscr{H}_{2} \rightarrow \mathscr{R} \cup\{+\infty\}$ are two proper, lower semicontinuous convex functions, and $A: \mathscr{H}_{1} \rightarrow \mathscr{H}_{2}$ is a bounded linear operator.

In the present paper, we are devoted to solving the following minimization problem:

$$
\min _{x^{\dagger} \in \mathscr{H}_{1}}\left\{f\left(x^{\dagger}\right)+g_{\lambda}\left(A x^{\dagger}\right)\right\}
$$

where $g_{\lambda}$ stands for the Moreau-Yosida approximation of the function $g$ of parameter $\lambda$; that is,

$$
g_{\lambda}(u)=\min _{v \in \mathscr{H}_{2}}\left\{g(v)+\frac{1}{2 \lambda}\|u-v\|^{2}\right\} .
$$

Problem (1) includes the split feasibility problem as a special case. In fact, we choose $f$ and $g$ as the indicator functions of two nonempty closed convex sets $C \subset \mathscr{H}_{1}$ and $Q \in \mathscr{H}_{2}$; that is,

$$
f\left(x^{\dagger}\right)=\delta_{C}\left(x^{\dagger}\right)= \begin{cases}0, & \text { if } x^{\dagger} \in C, \\ +\infty, & \text { otherwise, }\end{cases}
$$

$$
g\left(x^{\dagger}\right)=\delta_{\mathrm{Q}}\left(x^{\dagger}\right)= \begin{cases}0, & \text { if } x^{\dagger} \in Q \\ +\infty, & \text { otherwise }\end{cases}
$$

Then, problem (1) reduces to

$$
\min _{x^{\dagger} \in \mathscr{H}_{1}}\left\{\delta_{C}\left(x^{\dagger}\right)+\left(\delta_{\mathrm{Q}}\right)_{\lambda}\left(A x^{\dagger}\right)\right\},
$$

which equals

$$
\min _{x^{\dagger} \in C}\left\{\frac{1}{2 \lambda}\left\|\left(I-\operatorname{proj}_{Q}\right)\left(A x^{\dagger}\right)\right\|^{2}\right\} .
$$

Now we know that solving (5) is exactly to solve the following split feasibility problem of finding $x^{\ddagger}$ such that

$$
x^{\ddagger} \in C, \quad A x^{\ddagger} \in Q,
$$

provided $C \cap A^{-1}(Q) \neq \emptyset$.

The split feasibility problem in finite-dimensional Hilbert spaces was first introduced by Censor and Elfving [1] for modeling inverse problems which arise from phase retrievals and in medical image reconstruction. Recently, the split feasibility problem (6) has been studied extensively by many authors; see, for instance, [2-8]. 
In order to solve (6), one of the key ideas is to use fixed point technique according to $x^{\dagger}$ which solves (6) if and only if

$$
x^{\dagger}=\operatorname{proj}_{C}\left(I-\gamma A^{*}\left(I-\operatorname{proj}_{\mathrm{Q}}\right) A\right) x^{\dagger} .
$$

Next, we will use this idea to solve (1). First, by the differentiability of the Yosida approximation $g_{\lambda}$, we have

$$
\begin{gathered}
\partial\left(f\left(x^{\dagger}\right)+g_{\lambda}\left(A x^{\dagger}\right)\right)=\partial f\left(x^{\dagger}\right)+A^{*} \nabla g_{\lambda}\left(A x^{\dagger}\right) \\
=\partial f\left(x^{\dagger}\right)+A^{*}\left(\frac{I-\operatorname{prox}_{\lambda g}}{\lambda}\right)\left(A x^{\dagger}\right),
\end{gathered}
$$

where $\partial f\left(x^{\dagger}\right)$ denotes the subdifferential of $f$ at $x^{\dagger}$ and $\operatorname{prox}_{\lambda g}\left(x^{\dagger}\right)$ is the proximal mapping of $g$. That is,

$$
\begin{gathered}
\partial f\left(x^{\dagger}\right)=\left\{x^{*} \in \mathscr{H}_{1}: f\left(x^{\ddagger}\right) \geq f\left(x^{\dagger}\right)+\left\langle x^{*}, x^{\ddagger}-x^{\dagger}\right\rangle,\right. \\
\left.\forall x^{\ddagger} \in \mathscr{H}_{1}\right\}, \\
\operatorname{prox}_{\lambda g}\left(x^{\dagger}\right)=\arg \min _{x^{\ddagger} \in \mathscr{H}_{2}}\left\{g\left(x^{\ddagger}\right)+\frac{1}{2 \lambda}\left\|x^{\ddagger}-x^{\dagger}\right\|^{2}\right\} .
\end{gathered}
$$

Note that the optimality condition of (8) is as follows:

$$
0 \in \partial f\left(x^{\dagger}\right)+A^{*}\left(\frac{I-\operatorname{prox}_{\lambda g}}{\lambda}\right)\left(A x^{\dagger}\right),
$$

which can be rewritten as

$$
0 \in \mu \lambda \partial f\left(x^{\dagger}\right)+\mu A^{*}\left(I-\operatorname{prox}_{\lambda g}\right)\left(A x^{\dagger}\right),
$$

which is equivalent to the fixed point equation

$$
x^{\dagger}=\operatorname{prox}_{\mu \lambda f}\left(x^{\dagger}-\mu A^{*}\left(I-\operatorname{prox}_{\lambda g}\right)\right)\left(A x^{\dagger}\right) .
$$

If $\arg \min f \cap A^{-1}(\arg \min g) \neq \emptyset$, then (1) is reduced to the following proximal split feasibility problem of finding $x^{\dagger}$ such that

$$
x^{\dagger} \in \arg \min f, \quad A x^{\dagger} \in \arg \min g,
$$

where

$$
\begin{aligned}
& \arg \min f=\left\{x^{*} \in \mathscr{H}_{1}: f\left(x^{*}\right) \leq f\left(x^{\dagger}\right), \forall x^{\dagger} \in \mathscr{H}_{1}\right\}, \\
& \arg \min g=\left\{x^{\dagger} \in \mathscr{H}_{2}: g\left(x^{\dagger}\right) \leq g(x), \forall x \in \mathscr{H}_{2}\right\} .
\end{aligned}
$$

In the sequel, we will use $\Gamma$ to denote the solution set of (13).

Recently, in order to solve (13), Moudafi and Thakur [9] presented the following split proximal algorithm with a way of selecting the stepsizes such that its implementation does not need any prior information about the operator norm.

\section{Split Proximal Algorithm}

Step 1 (initialization).

$$
x_{0} \in \mathscr{H}_{1} \text {. }
$$

Step 2. Assume that $x_{n}$ has been constructed and $\theta\left(x_{n}\right) \neq \emptyset$. Then compute $x_{n+1}$ via the manner

$$
x_{n+1}=\operatorname{prox}_{\mu_{n} \lambda f}\left[x_{n}-\mu_{n} A^{*}\left(I-\operatorname{prox}_{\lambda g}\right) A x_{n}\right], \quad \forall n \geq 0,
$$

where the stepsize $\mu_{n}=\rho_{n}\left(\left(h\left(x_{n}\right)+l\left(x_{n}\right)\right) / \theta^{2}\left(x_{n}\right)\right)$ in which $0<\rho_{n}<4, h\left(x_{n}\right)=(1 / 2)\left\|\left(I-\operatorname{prox}_{\lambda g}\right) A x_{n}\right\|^{2}, l\left(x_{n}\right)=(1 /$ 2) $\left\|\left(I-\operatorname{prox}_{\mu_{n} \lambda f}\right) x_{n}\right\|^{2}$ and $\theta\left(x_{n}\right)=\sqrt{\left\|\nabla h\left(x_{n}\right)\right\|^{2}+\left\|\nabla l\left(x_{n}\right)\right\|^{2}}$.

If $\theta\left(x_{n}\right)=0$, then $x_{n+1}=x_{n}$ is a solution of (13) and the iterative process stops; otherwise, we set $n:=n+1$ and go to (16).

Consequently, they demonstrated the following weak convergence of the above split proximal algorithm.

Theorem 1. Suppose that $\Gamma \neq \emptyset$. Assume that the parameters satisfy the condition:

$\epsilon \leq \rho_{n} \leq \frac{4 h\left(x_{n}\right)}{h\left(x_{n}\right)+l\left(x_{n}\right)}-\epsilon$ for some $\epsilon>0$ small enough.

Then the sequence $x_{n}$ weakly converges to a solution of (13).

Note that the proximal mapping of $g$ is firmly nonexpansive, namely,

$$
\begin{array}{r}
\left\langle\operatorname{prox}_{\lambda g} x-\operatorname{prox}_{\lambda g} y, x-y\right\rangle \geq\left\|\operatorname{prox}_{\lambda g} x-\operatorname{prox}_{\lambda g} y\right\|^{2}, \\
\forall x, y \in \mathscr{H}_{2},
\end{array}
$$

and it is also the case for complement $I-\operatorname{prox}_{\lambda g}$. Thus, $A^{*}\left(I-\operatorname{prox}_{\lambda g}\right) A$ is cocoercive with coefficient $1 /\|A\|^{2}$ (recall that a mapping $B: \mathscr{H}_{1} \rightarrow \mathscr{H}_{1}$ is said to be cocoercive if $\langle B x-B y, x-y\rangle \geq \alpha\|B x-B y\|^{2}$ for all $x, y \in \mathscr{H}_{1}$ and some $\alpha>0)$. If $\mu \in\left(0,1 /\|A\|^{2}\right)$, then $I-\mu A^{*}\left(I-\operatorname{prox}_{\lambda q}\right) A$ is nonexpansive. Hence, we need to regularize (16) such that the strong convergence is obtained. This is the main purpose of this paper. In the next section, we will collect some useful lemmas and in the last section we will present our algorithm and prove its strong convergence.

\section{Lemmas}

Lemma 2 (see [10]). Let $\left\{a_{n}\right\}_{n \in \mathbb{N}}$ be a sequence of nonnegative real numbers satisfying the following relation:

$$
a_{n+1} \leq\left(1-\alpha_{n}\right) a_{n}+\alpha_{n} \sigma_{n}+\delta_{n}, \quad n \geq 0,
$$

where

(i) $\left\{\alpha_{n}\right\}_{n \in \mathbb{N}} \subset[0,1]$ and $\sum_{n=1}^{\infty} \alpha_{n}=\infty$;

(ii) $\lim \sup _{n \rightarrow \infty} \sigma_{n} \leq 0$;

(iii) $\sum_{n=1}^{\infty} \delta_{n}<\infty$.

Then, $\lim _{n \rightarrow \infty} a_{n}=0$. 
Lemma 3 (see [11]). Let $\left\{\gamma_{n}\right\}_{n \in \mathbb{N}}$ be a sequence of real numbers such that there exists a subsequence $\left\{\gamma_{n_{i}}\right\}_{i \in \mathbb{N}}$ of $\left\{\gamma_{n}\right\}_{n \in \mathbb{N}}$ such that $\gamma_{n_{i}}<\gamma_{n_{i}+1}$ for all $i \in \mathbb{N}$. Then, there exists a nondecreasing sequence $\left\{m_{k}\right\}_{k \in \mathbb{N}}$ of $\mathbb{N}$ such that $\lim _{k \rightarrow \infty} m_{k}=\infty$ and the following properties are satisfied by all (sufficiently large) numbers $k \in \mathbb{N}$ :

$$
\gamma_{m_{k}} \leq \gamma_{m_{k}+1}, \quad \gamma_{k} \leq \gamma_{m_{k}+1} .
$$

In fact, $m_{k}$ is the largest number $n$ in the set $\{1, \ldots, k\}$ such that the condition $\gamma_{n}<\gamma_{n+1}$ holds.

\section{Main results}

Let $\mathscr{H}_{1}$ and $\mathscr{H}_{2}$ be two real Hilbert spaces. Let $f: \mathscr{H}_{1} \rightarrow$ $\mathscr{R} \cup\{+\infty\}$ and $g: \mathscr{H}_{2} \rightarrow \mathscr{R} \cup\{+\infty\}$ be two proper, lower semicontinuous convex functions and $A: \mathscr{H}_{1} \rightarrow \mathscr{H}_{2}$ a bounded linear operator.

Now, we firstly introduce our algorithm.

\section{Algorithm 4}

Step 1 (initialization).

$$
x_{0} \in \mathscr{H}_{1} .
$$

Step 2. Assume that $x_{n}$ has been constructed. Set $h\left(x_{n}\right)=$ $(1 / 2)\left\|\left(I-\operatorname{prox}_{\lambda g}\right) A x_{n}\right\|^{2}, l\left(x_{n}\right)=(1 / 2)\left\|\left(I-\operatorname{prox}_{\mu_{n} \lambda f}\right) x_{n}\right\|^{2}$ and $\theta\left(x_{n}\right)=\sqrt{\left\|\nabla h\left(x_{n}\right)\right\|^{2}+\left\|\nabla l\left(x_{n}\right)\right\|^{2}}$ for all $n \in \mathbb{N}$.

If $\theta\left(x_{n}\right) \neq \emptyset$, then compute $x_{n+1}$ via the manner

$$
\begin{aligned}
& x_{n+1} \\
& =\operatorname{prox}_{\mu_{n} \lambda f}\left[\alpha_{n} u+\left(1-\alpha_{n}\right) x_{n}-\mu_{n} A^{*}\left(I-\operatorname{prox}_{\lambda g}\right) A x_{n}\right],
\end{aligned}
$$

where $u \in \mathscr{H}_{1}$ is a fixed point and $\left\{\alpha_{n}\right\}_{n \in \mathbb{N}} \subset[0,1]$ is a real number sequence and $\mu_{n}$ is the stepsize satisfying $\mu_{n}=$ $\rho_{n}\left(\left(h\left(x_{n}\right)+l\left(x_{n}\right)\right) / \theta^{2}\left(x_{n}\right)\right)$ with $0<\rho_{n}<4$.

If $\theta\left(x_{n}\right)=0$, then $x_{n+1}=x_{n}$ is a solution of (13) and the iterative process stops; otherwise, we set $n:=n+1$ and go to (22).

Theorem 5. Suppose that $\Gamma \neq \emptyset$. Assume that the parameters $\left\{\alpha_{n}\right\}$ and $\left\{\rho_{n}\right\}$ satisfy the conditions:

$$
\begin{aligned}
& \text { (C1) } \lim _{n \rightarrow \infty} \alpha_{n}=0 ; \\
& \text { (C2) } \sum_{n=0}^{\infty} \alpha_{n}=\infty \\
& \text { (C3) } \epsilon \leq \rho_{n} \leq\left(4 h\left(x_{n}\right) /\left(h\left(x_{n}\right)+l\left(x_{n}\right)\right)\right)-\epsilon \text { for some } \epsilon>0 \\
& \text { small enough. }
\end{aligned}
$$

Then the sequence $x_{n}$ converges strongly to $\operatorname{proj}_{\Gamma}(u)$.

Proof. Let $x^{*} \in \Gamma$. Since minimizers of any function are exactly fixed points of its proximal mappings, we have $x^{*}=\operatorname{prox}_{\mu_{n} \lambda f} x^{*}$ and $A x^{*}=\operatorname{prox}_{\lambda_{g}} A x^{*}$. By (22) and the nonexpansivity of $\operatorname{prox}_{\mu_{n} \lambda f}$, we derive

$$
\begin{aligned}
& \left\|x_{n+1}-x^{*}\right\|^{2} \\
& =\| \operatorname{prox}_{\mu_{n} \lambda f}\left[\alpha_{n} u+\left(1-\alpha_{n}\right) x_{n}-\mu_{n} A^{*}\left(I-\operatorname{prox}_{\lambda g}\right) A x_{n}\right] \\
& \quad-\operatorname{prox}_{\mu_{n} \lambda f} x^{*} \|^{2} \\
& \leq\left\|\alpha_{n} u+\left(1-\alpha_{n}\right) x_{n}-\mu_{n} A^{*}\left(I-\operatorname{prox}_{\lambda g}\right) A x_{n}-x^{*}\right\|^{2} \\
& =\| \alpha_{n}\left(u-x^{*}\right)+\left(1-\alpha_{n}\right) \\
& \quad \times\left[x_{n}-\frac{\mu_{n}}{1-\alpha_{n}} A^{*}\left(I-\operatorname{prox}_{\lambda g}\right) A x_{n}-x^{*}\right] \|^{2} \\
& \leq \alpha_{n}\left\|u-x^{*}\right\|^{2}+\left(1-\alpha_{n}\right) \\
& \quad \times\left\|x_{n}-\frac{\mu_{n}}{1-\alpha_{n}} A^{*}\left(I-\operatorname{prox}_{\lambda g}\right) A x_{n}-x^{*}\right\|^{2} .
\end{aligned}
$$

Since $\operatorname{prox}_{\lambda g}$ is firmly nonexpansive, we deduce that $I-\operatorname{prox}_{\lambda g}$ is also firmly nonexpansive. Hence, we have

$$
\begin{aligned}
& \left\langle A^{*}\left(I-\operatorname{prox}_{\lambda g}\right) A x_{n}, x_{n}-x^{*}\right\rangle \\
& \quad=\left\langle\left(I-\operatorname{prox}_{\lambda g}\right) A x_{n}, A x_{n}-A x^{*}\right\rangle \\
& =\left\langle\left(I-\operatorname{prox}_{\lambda g}\right) A x_{n}-\left(I-\operatorname{prox}_{\lambda g}\right) A x^{*}, A x_{n}-A x^{*}\right\rangle \\
& \geq\left\|\left(I-\operatorname{prox}_{\lambda g}\right) A x_{n}\right\|^{2}=2 h\left(x_{n}\right) .
\end{aligned}
$$

Note that $\nabla h\left(x_{n}\right)=A^{*}\left(I-\operatorname{prox}_{\lambda g}\right) A x_{n}$ and $\nabla l\left(x_{n}\right)=(I-$ $\left.\operatorname{prox}_{\mu_{n} \lambda f}\right) x_{n}$. From (24), we obtain

$$
\begin{aligned}
& \left\|x_{n}-\frac{\mu_{n}}{1-\alpha_{n}} A^{*}\left(I-\operatorname{prox}_{\lambda g}\right) A x_{n}-x^{*}\right\|^{2} \\
& =\left\|x_{n}-x^{*}\right\|^{2}+\frac{\mu_{n}^{2}}{\left(1-\alpha_{n}\right)^{2}}\left\|A^{*}\left(I-\operatorname{prox}_{\lambda g}\right) A x_{n}\right\|^{2} \\
& \quad-\frac{2 \mu_{n}}{1-\alpha_{n}}\left\langle A^{*}\left(I-\operatorname{prox}_{\lambda g}\right) A x_{n}, x_{n}-x^{*}\right\rangle \\
& =\left\|x_{n}-x^{*}\right\|^{2}+\frac{\mu_{n}^{2}}{\left(1-\alpha_{n}\right)^{2}}\left\|\nabla h\left(x_{n}\right)\right\|^{2} \\
& \quad-\frac{2 \mu_{n}}{1-\alpha_{n}}\left\langle\nabla h\left(x_{n}\right), x_{n}-x^{*}\right\rangle \\
& \leq \\
& \quad\left\|x_{n}-x^{*}\right\|^{2}+\frac{\mu_{n}^{2}}{\left(1-\alpha_{n}\right)^{2}}\left\|\nabla h\left(x_{n}\right)\right\|^{2} \\
& \quad-\frac{4 \mu_{n} h\left(x_{n}\right)}{1-\alpha_{n}}
\end{aligned}
$$




$$
\begin{aligned}
= & \left\|x_{n}-x^{*}\right\|^{2}+\rho_{n}^{2} \frac{\left(h\left(x_{n}\right)+l\left(x_{n}\right)\right)^{2}}{\left(1-\alpha_{n}\right)^{2} \theta^{4}\left(x_{n}\right)}\left\|\nabla h\left(x_{n}\right)\right\|^{2} \\
& -4 \rho_{n} \frac{h\left(x_{n}\right)+l\left(x_{n}\right)}{\left(1-\alpha_{n}\right) \theta^{2}\left(x_{n}\right)} h\left(x_{n}\right) \\
\leq & \left\|x_{n}-x^{*}\right\|^{2}+\rho_{n}^{2} \frac{\left(h\left(x_{n}\right)+l\left(x_{n}\right)\right)^{2}}{\left(1-\alpha_{n}\right)^{2} \theta^{2}\left(x_{n}\right)} \\
& -4 \rho_{n} \frac{\left(h\left(x_{n}\right)+l\left(x_{n}\right)\right)^{2}}{\left(1-\alpha_{n}\right) \theta^{2}\left(x_{n}\right)} \frac{h\left(x_{n}\right)}{h\left(x_{n}\right)+l\left(x_{n}\right)} \\
= & \left\|x_{n}-x^{*}\right\|^{2}-\rho_{n}\left(\frac{4 h\left(x_{n}\right)}{h\left(x_{n}\right)+l\left(x_{n}\right)}-\frac{\rho_{n}}{1-\alpha_{n}}\right) \\
& \times \frac{\left(h\left(x_{n}\right)+l\left(x_{n}\right)\right)^{2}}{\left(1-\alpha_{n}\right) \theta^{2}\left(x_{n}\right)} .
\end{aligned}
$$

By condition (C3), without loss of generality, we can assume that $\left(4 h\left(x_{n}\right) /\left(h\left(x_{n}\right)+l\left(x_{n}\right)\right)\right)-\left(\rho_{n} /\left(1-\alpha_{n}\right)\right) \geq 0$ for all $n \geq 0$. Thus, from (23) and (25), we obtain

$$
\begin{aligned}
& \left\|x_{n+1}-x^{*}\right\|^{2} \\
& \leq \alpha_{n}\left\|u-x^{*}\right\|^{2}+\left(1-\alpha_{n}\right) \\
& \quad \times\left[\left\|x_{n}-x^{*}\right\|^{2}\right. \\
& \left.\quad-\rho_{n}\left(\frac{4 h\left(x_{n}\right)}{h\left(x_{n}\right)+l\left(x_{n}\right)}-\frac{\rho_{n}}{1-\alpha_{n}}\right) \frac{\left(h\left(x_{n}\right)+l\left(x_{n}\right)\right)^{2}}{\left(1-\alpha_{n}\right) \theta^{2}\left(x_{n}\right)}\right] \\
& =\alpha_{n}\left\|u-x^{*}\right\|^{2}+\left(1-\alpha_{n}\right)\left\|x_{n}-x^{*}\right\|^{2} \\
& \quad-\rho_{n}\left(\frac{4 h\left(x_{n}\right)}{h\left(x_{n}\right)+l\left(x_{n}\right)}-\frac{\rho_{n}}{1-\alpha_{n}}\right) \frac{\left(h\left(x_{n}\right)+l\left(x_{n}\right)\right)^{2}}{\theta^{2}\left(x_{n}\right)} \\
& \leq \alpha_{n}\left\|u-x^{*}\right\|^{2}+\left(1-\alpha_{n}\right)\left\|x_{n}-x^{*}\right\|^{2} \\
& \leq \max \left\{\left\|u-x^{*}\right\|^{2},\left\|x_{n}-x^{*}\right\|^{2}\right\} .
\end{aligned}
$$

Hence, $\left\{x_{n}\right\}$ is bounded.

Let $z=P_{\Gamma} u$. From (26), we deduce

$$
\begin{aligned}
0 & \leq \rho_{n}\left(\frac{4 h\left(x_{n}\right)}{h\left(x_{n}\right)+l\left(x_{n}\right)}-\frac{\rho_{n}}{1-\alpha_{n}}\right) \frac{\left(h\left(x_{n}\right)+l\left(x_{n}\right)\right)^{2}}{\theta^{2}\left(x_{n}\right)} \\
& \leq \alpha_{n}\|u-z\|^{2}+\left(1-\alpha_{n}\right)\left\|x_{n}-z\right\|^{2}-\left\|x_{n+1}-z\right\|^{2} .
\end{aligned}
$$

We consider the following two cases.

Case 1. One has $\left\|x_{n+1}-z\right\| \leq\left\|x_{n}-z\right\|$ for every $n \geq n_{0}$ large enough.

In this case, $\lim _{n \rightarrow \infty}\left\|x_{n}-z\right\|$ exists as finite and hence

$$
\lim _{n \rightarrow \infty}\left(\left\|x_{n+1}-z\right\|-\left\|x_{n}-z\right\|\right)=0 .
$$

This together with (27) implies that

$$
\rho_{n}\left(\frac{4 h\left(x_{n}\right)}{h\left(x_{n}\right)+l\left(x_{n}\right)}-\frac{\rho_{n}}{1-\alpha_{n}}\right) \frac{\left(h\left(x_{n}\right)+l\left(x_{n}\right)\right)^{2}}{\theta^{2}\left(x_{n}\right)} \longrightarrow 0 .
$$

Since $\liminf _{n \rightarrow \infty} \rho_{n}\left(\left(4 h\left(x_{n}\right) /\left(h\left(x_{n}\right)+l\left(x_{n}\right)\right)\right)-\left(\rho_{n} /\left(1-\alpha_{n}\right)\right)\right) \geq$ $2 \epsilon$ (by condition (C3)), we get

$$
\frac{\left(h\left(x_{n}\right)+l\left(x_{n}\right)\right)^{2}}{\theta^{2}\left(x_{n}\right)} \longrightarrow 0 .
$$

Noting that $\theta^{2}\left(x_{n}\right)=\left\|\nabla h\left(x_{n}\right)\right\|^{2}+\left\|\nabla l\left(x_{n}\right)\right\|^{2}$ is bounded, we deduce immediately that

$$
\lim _{n \rightarrow \infty}\left(h\left(x_{n}\right)+l\left(x_{n}\right)\right)=0 .
$$

Therefore,

$$
\lim _{n \rightarrow \infty} h\left(x_{n}\right)=\lim _{n \rightarrow \infty} l\left(x_{n}\right)=0 .
$$

Next, we prove

$$
\limsup _{n \rightarrow \infty}\left\langle u-z, x_{n}-z\right\rangle \leq 0 .
$$

Since $\left\{x_{n}\right\}$ is bounded, there exists a subsequence $\left\{x_{n_{i}}\right\}$ satisfying $x_{n_{i}} \rightarrow z^{\dagger}$ and

$$
\limsup _{n \rightarrow \infty}\left\langle u-z, x_{n}-z\right\rangle=\lim _{i \rightarrow \infty}\left\langle u-z, x_{n_{i}}-z\right\rangle .
$$

By the lower semicontinuity of $h$, we get

$$
0 \leq h\left(z^{\dagger}\right) \leq \liminf _{i \rightarrow \infty} h\left(x_{n_{i}}\right)=\lim _{n \rightarrow \infty} h\left(x_{n}\right)=0 .
$$

So,

$$
h\left(z^{\dagger}\right)=\frac{1}{2}\left\|\left(I-\operatorname{prox}_{\lambda g}\right) A z^{\dagger}\right\|=0 .
$$

That is, $A z^{\dagger}$ is a fixed point of the proximal mapping of $g$ or equivalently $0 \in \partial g\left(A z^{\dagger}\right)$. In other words, $A z^{\dagger}$ is a minimizer of $g$.

Similarly, from the lower semicontinuity of $l$, we get

$$
0 \leq l\left(z^{\dagger}\right) \leq \liminf _{i \rightarrow \infty} l\left(x_{n_{i}}\right)=\lim _{n \rightarrow \infty} l\left(x_{n}\right)=0 .
$$

Therefore,

$$
l\left(z^{\dagger}\right)=\frac{1}{2}\left\|\left(I-\operatorname{prox}_{\mu_{n} \lambda f}\right) z^{\dagger}\right\|=0 .
$$

That is, $z^{\dagger}$ is a fixed point of the proximal mapping of $f$ or equivalently $0 \in \partial f\left(z^{\dagger}\right)$. In other words, $z^{\dagger}$ is a minimizer of $f$. Hence, $z^{\dagger} \in \Gamma$. Therefore,

$$
\begin{aligned}
\limsup _{n \rightarrow \infty}\left\langle u-z, x_{n}-z\right\rangle & =\lim _{i \rightarrow \infty}\left\langle u-z, x_{n_{i}}-z\right\rangle \\
& =\left\langle u-z, z^{\dagger}-z\right\rangle \leq 0 .
\end{aligned}
$$


From (22), we have

$$
\begin{aligned}
& \left\|x_{n+1}-z\right\|^{2} \\
& \leq \| \alpha_{n}(u-z)+\left(1-\alpha_{n}\right) \\
& \quad \times\left[x_{n}-\frac{\mu_{n}}{1-\alpha_{n}} A^{*}\left(I-\operatorname{prox}_{\lambda g}\right) A x_{n}-z\right] \|^{2} \\
& =\left(1-\alpha_{n}\right)^{2}\left\|x_{n}-\frac{\mu_{n}}{1-\alpha_{n}} A^{*}\left(I-\operatorname{prox}_{\lambda g}\right) A x_{n}-z\right\|^{2} \\
& \quad+\alpha_{n}^{2}\|u-z\|^{2}+2 \alpha_{n}\left(1-\alpha_{n}\right) \\
& \quad \times\left\langle x_{n}-\frac{\mu_{n}}{1-\alpha_{n}} A^{*}\left(I-\operatorname{prox}_{\lambda g}\right) A x_{n}-z, u-z\right\rangle \\
& \leq\left(1-\alpha_{n}\right)^{2}\left\|x_{n}-z\right\|^{2}+\alpha_{n}^{2}\|u-z\|^{2} \\
& +2 \alpha_{n}\left(1-\alpha_{n}\right)\left\langle x_{n}-z, u-z\right\rangle \\
& \quad-2 \alpha_{n} \mu_{n}\left\langle\nabla h\left(x_{n}\right), u-z\right\rangle \\
& \leq\left(1-\alpha_{n}\right)\left\|x_{n}-z\right\|^{2} \\
& +\alpha_{n}\left(\alpha_{n}\|u-z\|^{2}+2\left(1-\alpha_{n}\right)\left\langle x_{n}-z, u-z\right\rangle\right. \\
& \left.\quad+2 \mu_{n}\left\|\nabla h\left(x_{n}\right)\right\|\|u-z\|\right) .
\end{aligned}
$$

Since $\nabla h$ is Lipschitz continuous with Lipschitzian constant $\|A\|^{2}$ and $\nabla l$ is nonexpansive, $\nabla h\left(u_{n}\right), \nabla l\left(u_{n}\right)$, and $\theta^{2}\left(x_{n}\right)=$ $\left\|\nabla h\left(x_{n}\right)\right\|^{2}+\left\|\nabla l\left(x_{n}\right)\right\|^{2}$ are bounded. Note that $\mu_{n}\left\|\nabla h\left(x_{n}\right)\right\|=$ $\rho_{n}\left(\left(h\left(x_{n}\right)+l\left(x_{n}\right)\right) / \theta^{2}\left(x_{n}\right)\right)\left\|\nabla h\left(x_{n}\right)\right\|$. Thus, $\mu_{n}\left\|\nabla h\left(x_{n}\right)\right\| \rightarrow 0$ by (32). From Lemma 2, (39), and (40) we deduce that $x_{n} \rightarrow$ $z$.

Case 2. There exists a subsequence $\left\{\left\|x_{n_{j}}-z\right\|\right\}$ of $\left\{\left\|x_{n}-z\right\|\right\}$ such that

$$
\left\|x_{n_{j}}-z\right\|<\left\|x_{n_{j}+1}-z\right\|
$$

for all $j \geq 1$. By Lemma 3, there exists a strictly increasing sequence $\left\{m_{k}\right\}$ of positive integers such that $\lim _{k \rightarrow \infty} m_{k}=$ $+\infty$ and the following properties are satisfied by all numbers $k \in \mathbb{N}$ :

$$
\left\|x_{m_{k}}-z\right\| \leq\left\|x_{m_{k+1}}-z\right\|, \quad\left\|x_{k}-z\right\| \leq\left\|x_{m_{k+1}}-z\right\| .
$$

Consequently,

$$
\begin{aligned}
0 & \leq \lim _{k \rightarrow \infty}\left(\left\|x_{m_{k+1}}-z\right\|-\left\|x_{m_{k}}-z\right\|\right) \\
& \leq \limsup _{n \rightarrow \infty}\left(\left\|x_{n+1}-z\right\|-\left\|x_{n}-z\right\|\right) \\
& \leq \limsup _{n \rightarrow \infty}\left(\alpha_{n}\|u-z\|+\left(1-\alpha_{n}\right)\left\|x_{n}-z\right\|-\left\|x_{n}-z\right\|\right) \\
& =\limsup _{n \rightarrow \infty} \alpha_{n}\left(\|u-z\|-\left\|x_{n}-z\right\|\right)=0 .
\end{aligned}
$$

Hence,

$$
\lim _{k \rightarrow \infty}\left(\left\|x_{m_{k+1}}-z\right\|-\left\|x_{m_{k}}-z\right\|\right)=0 .
$$

By a similar argument as that of Case 1, we can prove that

$$
\begin{gathered}
\limsup _{k \rightarrow \infty}\left\langle u-z, x_{m_{k}}-z\right\rangle \leq 0, \\
\left\|x_{m_{k}+1}-z\right\|^{2} \leq\left(1-\alpha_{m_{k}}\right)\left\|x_{m_{k}}-z\right\|^{2}+\alpha_{m_{k}} \sigma_{m_{k}},
\end{gathered}
$$

where $\sigma_{m_{k}}=\alpha_{m_{k}}\|u-z\|^{2}+2\left(1-\alpha_{m_{k}}\right)\left\langle x_{m_{k}}-z, u-z\right\rangle+$ $2 \mu_{m_{k}}\left\|\nabla h\left(x_{m_{k}}\right)\right\|\|u-z\|$.

In particular, we get

$$
\begin{aligned}
& \alpha_{m_{k}}\left\|x_{m_{k}}-z\right\|^{2} \\
& \leq\left\|x_{m_{k}}-z\right\|^{2}-\left\|x_{m_{k}+1}-z\right\|+\alpha_{m_{k}} \sigma_{m_{k}} \\
& \leq \alpha_{m_{k}} \sigma_{m_{k}} .
\end{aligned}
$$

Then,

$$
\limsup _{k \rightarrow \infty}\left\|x_{m_{k}}-z\right\|^{2} \leq \limsup _{k \rightarrow \infty} \sigma_{m_{k}} \leq 0 .
$$

Thus, from (42) and (44), we conclude that

$$
\underset{k \rightarrow \infty}{\limsup }\left\|x_{k}-z\right\| \leq \underset{k \rightarrow \infty}{\limsup _{k}\left\|x_{m_{k}+1}-z\right\|=0 .}
$$

Therefore, $x_{n} \rightarrow z$. This completes the proof.

Remark 6. Note that problem (13) was considered, for example, in $[12,13]$; however, the iterative methods proposed to solve it need to know a priori the norm of the bounded linear operator $A$.

Remark 7. We would like also to emphasize that by taking $f=$ $\delta_{C}, g=\delta_{\mathrm{Q}}$ the indicator functions of two nonempty closed convex sets $C, Q$ of $H_{1}$ and $H_{2}$ respectively, our algorithm (22) reduces to

$$
\begin{aligned}
& x_{n+1} \\
& \quad=\operatorname{proj}_{C}\left[\alpha_{n} u+\left(1-\alpha_{n}\right) x_{n}-\mu_{n} A^{*}\left(I-\operatorname{proj}_{\mathrm{Q}}\right) A x_{n}\right],
\end{aligned}
$$$$
\forall n \geq 0 .
$$

We observe that (49) is simpler than the one in [14].

\section{Conflict of Interests}

The authors declare that there is no conflict of interests regarding the publication of this paper.

\section{Acknowledgments}

The authors are grateful to the referees for their valuable comments and suggestions. Sun Young Cho was supported 
by the Basic Science Research Program through the National Research Foundation of Korea funded by the Ministry of Education, Science and Technology (KRF-2013053358). LiJun Zhu was supported in part by NNSF of China (61362033 and NZ13087).

\section{References}

[1] Y. Censor and T. Elfving, "A multiprojection algorithm using Bregman projections in a product space," Numerical Algorithms, vol. 8, no. 2-4, pp. 221-239, 1994.

[2] C. Byrne, "Iterative oblique projection onto convex sets and the split feasibility problem," Inverse Problems, vol. 18, no. 2, pp. 441453, 2002.

[3] H.-K. Xu, "Iterative methods for the split feasibility problem in infinite-dimensional Hilbert spaces," Inverse Problems, vol. 26, no. 10, Article ID 105018, 17 pages, 2010.

[4] C. Byrne, "A unified treatment of some iterative algorithms in signal processing and image reconstruction," Inverse Problems, vol. 20, no. 1, pp. 103-120, 2004.

[5] J. Zhao and Q. Yang, "Several solution methods for the split feasibility problem," Inverse Problems, vol. 21, no. 5, pp. 17911799, 2005.

[6] Y. Dang and Y. Gao, "The strong convergence of a KM-CQ-like algorithm for a split feasibility problem," Inverse Problems, vol. 27, no. 1, Article ID 015007, 9 pages, 2011.

[7] F. Wang and $\mathrm{H}$. Xu, "Approximating curve and strong convergence of the CQ algorithm for the split feasibility problem," Journal of Inequalities and Applications, vol. 2010, Article ID 102085, 13 pages, 2010.

[8] Y. Yao, W. Jigang, and Y. Liou, "Regularized methods for the split feasibility problem," Abstract and Applied Analysis, vol. 2012, Article ID 140679, 13 pages, 2012.

[9] A. Moudafi and B. S. Thakur, "Solving proximal split feasibility problems without prior knowledge of operator norms," Optimization Letters, 2013.

[10] H. K. Xu, "Iterative algorithms for nonlinear operators," Journal of the London Mathematical Society, vol. 66, no. 1, pp. 240-256, 2002.

[11] P. E. Maingé, "Strong convergence of projected subgradient methods for non-smooth and non-strictly convex minimization," Set-Valued Analysis, vol. 16, no. 7-8, pp. 899-912, 2008.

[12] A. Moudafi, "Split monotone variational inclusions," Journal of Optimization Theory and Applications, vol. 150, no. 2, pp. 275283, 2011.

[13] C. Byrne, Y. Censor, A. Gibaliand, and S. Reich, "Weak and strong convergence of algorithms for the split common null point problem," Journal of Nonlinear and Convex Analysis, vol. 13, pp. 759-775, 2012.

[14] Y. Yu, "An explicit method for the split feasibility problem with self-adaptive step sizes," Abstract and Applied Analysis, vol. 2012, Article ID 432501, 9 pages, 2012. 


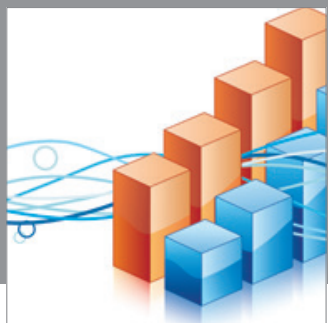

Advances in

Operations Research

mansans

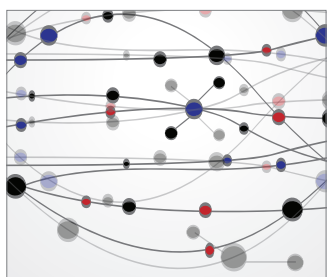

The Scientific World Journal
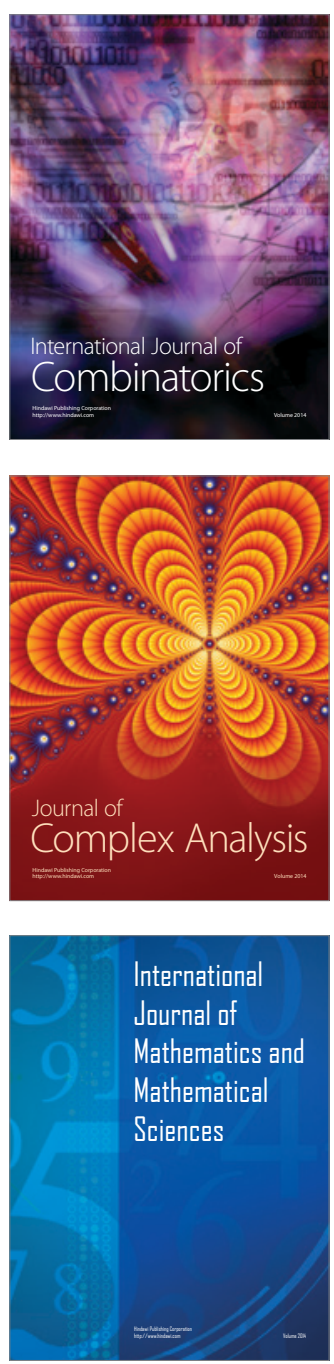
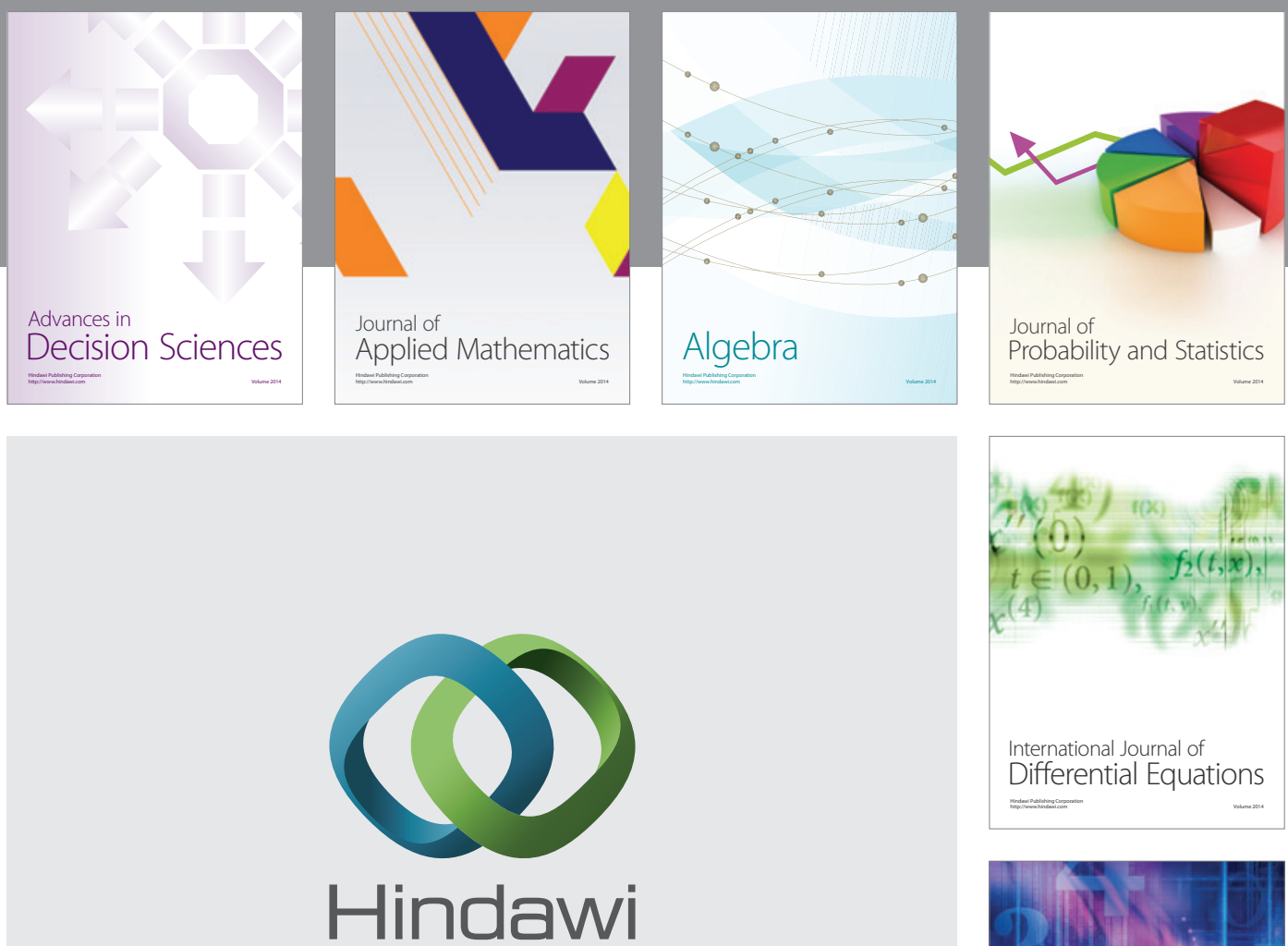

Submit your manuscripts at http://www.hindawi.com
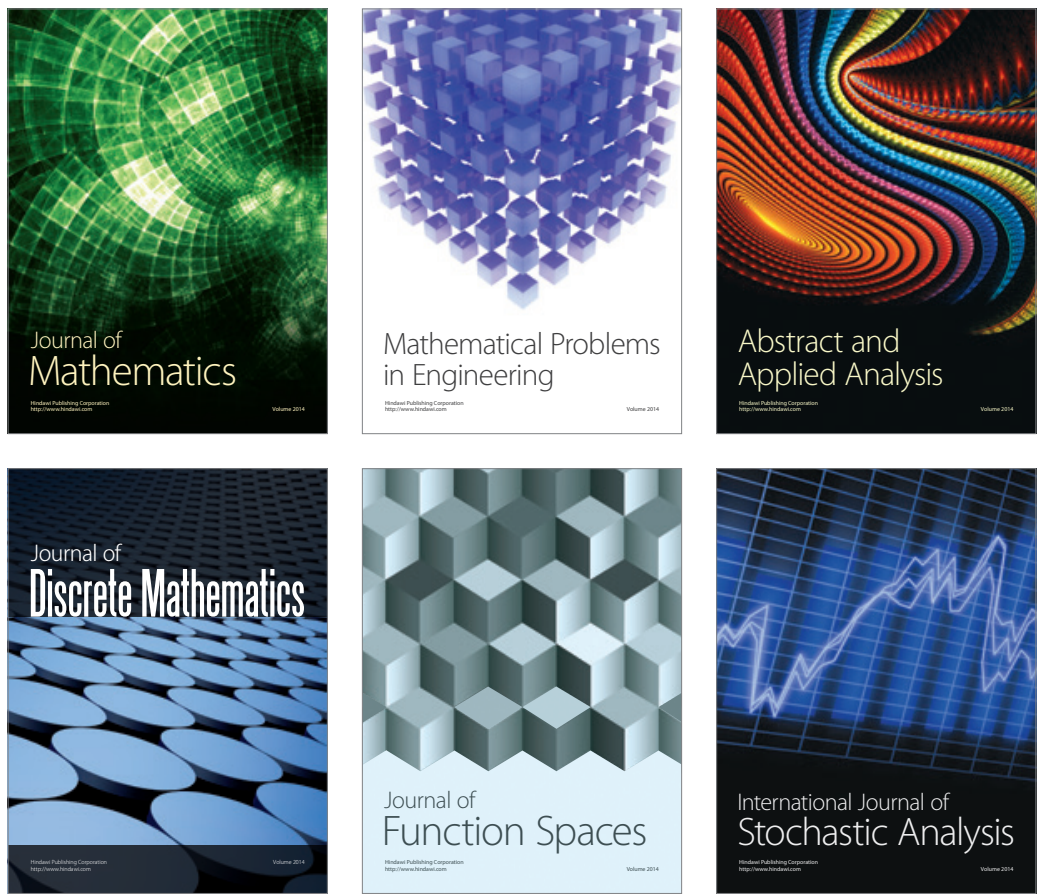

Journal of

Function Spaces

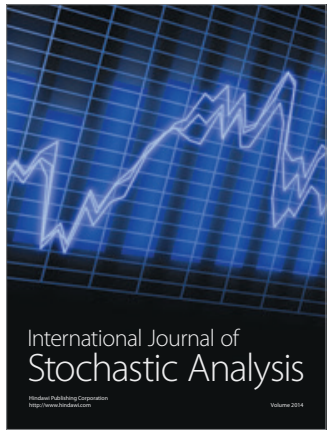

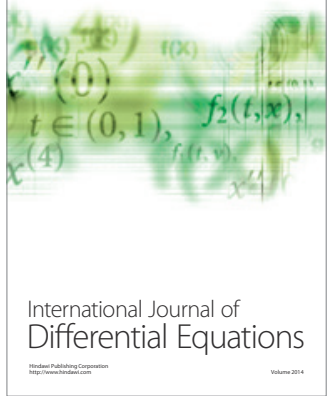
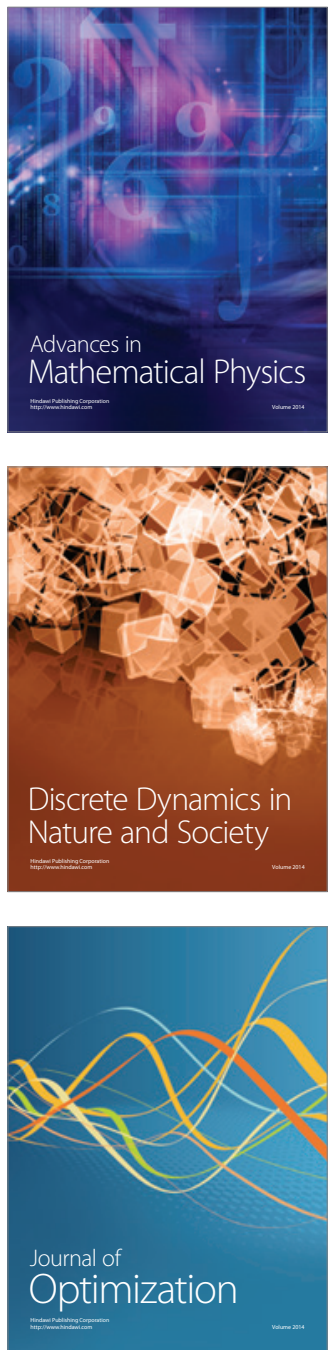